Ann. Abeille, I963, 6 (4), 303-320.

\title{
ÉTUDE DE LA TRANSLATION DU POINT DISCOÏDAL (DISCOID ALVERSCHIEBUNG) DE L'AILE DE L'ABEILLE (A. MELLIFICA L.)
}

\author{
I. LOLIS \\ Station de Recherches sur l'Abille et les Insectes sociaux, \\ Bures-sur-Yvette (Seine-et-Oise)
}

SOMMAIRE

Le point discoïdal de l'aile est soumis $\vdots a$ un type de fluctuation bien défini. Les aspects de cette variation sont considérés comme des caractères relatifs de subspéciation de l'Abeille domestique. L'auteur, après une étude de ces fluctuations, tente d'en apporter une interprétation graphique, dans le but de mettre en évidence les aspects cinétiques de ce caractère. 11 termine par une étude critique de la méthode.

\section{IN'TRODUC'TION}

Ce premier travail s'inscrit dans le cadre d'une série de publications, dont le but est de rechercher et de prouver l'existence d'éventuelles populations locales parmi 1'Abeille française. La connaissance des caractéristiques morphologiques régionales des colonies, doit permettre par la suite de confirmer la consanguinité des lignées lors d'éventuels travaux de sélection portant sur l'amélioration du rendement du cheptel.

La discrimination de formes diverses au sein d'une espèce est subordonnée à la connaissance de la variation de chacun des caractères susceptibles de distinguer chacune de ces formes. Les fluctuations du point discoïdal de l'aile constituent le premier caractère alaire que nous ayons étudié dans le but de préciser l'aspect de cette variation.

La bibliographie du sujet est pauvre. C'est Gokrze (I959) qui a le premier donné la description de ce caractère et a tenté son utilisation. Les quelques auteurs qui ont étudié la morphologie des "races " d'abeilles ou des soit-disant "écotypes " et notamment Aipatov, Rutrixer, Sakagami, etc. n'en ont pas fait mention dans leurs 
travaux. Il n'est cependant pas possible de relever par ailleurs des arguments permettant de l'abandonner.

Cette étude ne présente pas seulement un intérêt agronomique. Il est apparu en cours de travaux que les résultats pouvaient être mis en parallèle avec ceux des auteurs ayant publié en systématique évolutive (selon le sens donné à ce mot par BOQUET, I953). De même qu'il existe des caractères spécifiques, subspécifiques ou génériques, la translation discoïdale nous apparaît comme un caractère exergique tel que l'exerge a été décrit par VERITY (1925) et repris récemment dans la systématique française par BERNARDI (I957). C'est ainsi que la translation discoïdale dite positive semble être le propre d'un groupe de sous-espèces géographiquement voisines et constamment importées en France.

La première partie qui comprend l'exposé des résultats et la discussion correspondante, porte plus particulièrement sur la description de la variation géographique du caractère et la mise en évidence de clines (selon le sens donné à ce mot par HUXLEY (r938). La seconde partie de la discussion ne se situe plus au niveau des populations, mais on y compare la variation de la morphologie alaire entre individus de types opposés.

Les résultats et la discussion ne portent pas seulement sur l'Abeille française. 'Toutefois, celle-ci constitue la majeure partie de notre étude. Des échantillons en provenance d'Iispagne, d'Italie, d'Afrique du Nord et du Liban permettent de comparer les écarts ou les modifications progressives du caractère et de situer géographiquement les limites et les différents aspects des fluctuations auxquelles il est soumis.

\section{MATÉRIEL, ET MÉTHODES}

\section{A) Matiriel}

l.es échantillons proviennent de la plupart des grandes régions géographiques françaises. Trentecinc correspondants ont bien voulu nous adresser le natériel nécessaire qui porte sur trois cents ruches. Neuf mille abeilles ont été examinées sous cinq aspects différents, c'est donc au total 45 ooo données qui ont été classées et interprétées. La présente note ne porte que sur une partie de ces données, soit 6412 abeilles sont $545^{2}$ pour la France. Le reste de l'échantillonnage, soit 960 abeilles, se répartit entre l'Espagne, l'Al lgérie, la Tunisie, le Maroc et le Liban. Les méthodes de récolte et d $\mathbf{d}$ conservation ont été exposées dans une précédente publication ${ }^{(1)}$ à laquelle pour plus de détails on voudra bien se repurter.

\section{B) Méthodes}

I") Montage des ailes : Après dissection, les ailes ont été montées à sec entre deux lames minces jointées aux quatre angles par des points de pâte à modeler.

$2^{\circ}$ ) Les obseriations portant sur ia translation discoïdale ont été effectuées is la loupe binoculaire objectif $\times$ I ; oculaire $\times 25$. Ce dernier était équipé d'une réticule oculaire.

$\left.3^{\circ}\right)$ Les dessins ont été réalisés ì partir des mêmes préparations par projection sur papier de la silhouette de l'aile a l'aide d'un agrandisseur photographique (grossissement environ $\times 3^{\circ}$ ).

\section{() Description du caractère morphologique}

Cette description est empruntée à Golstze (1960) figure i.

$\mathrm{I}^{\circ)}$ On joint les extrémités de la cellule radiale par une droite $A B$;

$2^{0}$ ) par le point $\mathrm{C}$, on abaisse la perpendiculaire à cette droit $\mathrm{Y}^{\prime}$;

(1) Variation des associations polliniques observées dans des miels récoltés en un même lieu par des abeilles appartenant à des sous-espèces diffirentes. Anm. Abeille, $1963,11^{\circ} 3$. 
$3^{\circ}$ ) on observe la situation du point U par rapport ia cette perpendiculaire. Il peut alors, par rapport à celle-ci, prendre trois positions :

a) le point U peut être situé vers l'attache de l'aile, la translation discoïdale est dite alors négative.

b) ce point peut être situé sur l'axe vertical. La translation est alors considéré comme mulle.

c) le point U peut se situer au-delà de la droite yy'vers l'ajex de l'aile, la translation dans ce cas est dite posilive.

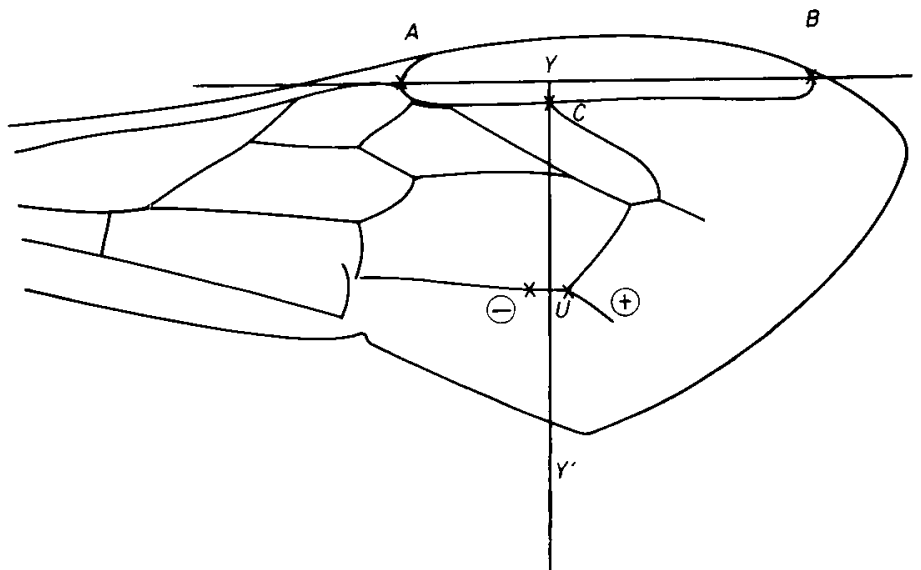

Fit, I. - Détermination d'un type de fluctuation discoidale positive sur une aile

t'A. mellifica ligustica SPIN et construction des axes de référence

Chaque point de convergence des nervures est utilisé comme repère initial, les lignes n'ont pour but que de reconstituer la silhouette de l'aile.

\section{RÉSUL'TATS}

\section{A. - ExPOSÉ DES RÉSUltats FT TABr.EAUX}

\section{$\left.\mathrm{I}^{0}\right)$ France}

Les résultats ont été recueillis selon la méthode classique. Les données ont été réunies, pour l'ensemble du territoire français, dans le tableau $\mathrm{I}$.

Informations complémentaires au tableau I. - Dans la présente note, seule la translation discoïdale est considérée, mais d'après une étude comparée avec quatre autres caractères, nous avons acquis la certitude que :

a) en ce qui concerne la région des Landes, le pourcentage de 16,58 correspondant aux positions positives est influencé en partie par des importations récentes d'abeilles italiennes (A.m. ligustica Spin). Il en est de même pour la région de la Vallée de la Loire ;

b) le pourcentage de II, 3o relatif à la région du Massif Central est influencé par la présence dans l'un des ruchers qui compose l'échantillon d'une forme quadrihybride américaine de type italien dominant;

c) le pourcentage de II,33 relatif à la région "Alsace-Lorraine " est influencé par des formes carnioliennes (A.m. carnica) couramment importées dans les régions de l'ist et en Allenagne.

Toutes ces influences de populations créées à partir de reines artificiellement émigrées seront plus amplement prouvées lors de publications ultérieures. Ces infor- 
TABLEAU I

Tableau des pourcentages relevés dans différcntes régions de Firance pour chacun des trois types de fluctuation du caractère. L'échantillon nommé "Bretagne" a été divisé en deux parties. On considérera que la première ligne (Bretagne I) correspond à une population existant en Bretagne. La seconde ligne est relative à des échantillons prélevés à Rennes, Laval et Le .Mans. Le regroupement de ces deux régions sous le même zocable tend à désigner une zone biogíographique oi les ćchantillons présentent par ailleurs certaines affinités entre cux

\begin{tabular}{|c|c|c|c|c|}
\hline \multirow{2}{*}{ Kérions } & \multicolumn{3}{|c|}{ en pourcentage } & \multirow{2}{*}{$\begin{array}{l}\text { Nombre } \\
\text { d'abeilles }\end{array}$} \\
\hline & $\perp$ & () & - & \\
\hline Bassin parisien . ....... & $5,8=$ & $(i 2,9 ;)$ & 28,23 & 510 \\
\hline Normandie ..... & 3,97 & $19,2: 3$ & 16,80 & $8:-27$ \\
\hline Bretagne $1 \ldots$ & 6.73 & $6: 3,77^{\prime}$ & $\because 9,5: 3$ & 150 \\
\hline Bretarne $\because \ldots \ldots \ldots \ldots$ & 3,12 & 52,11 & 科, & 535 \\
\hline 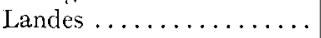 & 16,58 & 55,12 & 28,00 & $1 \div 00$ \\
\hline Massif Central .......... & 11,30 & 51,07 & $3 / 4,63$ & 540 \\
\hline Vallée de la Loire ....... & 20,00 & $47,3: 3$ & 32.67 & 50 \\
\hline Midi méditerranéen ...... & 3,78 & $59,3: 3$ & 36,89 & 150 \\
\hline Jura $\ldots \ldots \ldots \ldots \ldots \ldots$ & 5,36 & $7 x, 00$ & $20,{ }^{\prime} t_{t}^{\prime}$ & 150 \\
\hline Alpes ................ & 2,08 & 79,58 & $18,33_{1}^{\prime}$ & 240 \\
\hline Alsace-Lorraine $\ldots \ldots \ldots$ & 11,33 & 75,67 & 13,00 & 300 \\
\hline Pourcentagres moyens .... & 9,89 & 59,56 & 30,55 & \\
\hline 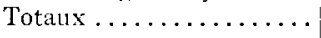 & & & & 5452 \\
\hline
\end{tabular}

mations complémentaires n'ont pout objet que d'expliquer l'existence dans la catégorie positive de pourcentages supérieurs ou égaux à Io p. IOo, par l'influence de sousespèces non indigènes.

Si l'on résume la totalité de l'information apportée par la tableau $\mathrm{n}^{\circ} \mathrm{I}$, on note que les pourcentages généraux arrondis à I p. Ioo près et relatifs à chacune des trois catégories sont nettement Io p. IOO, $60 \mathrm{p}$. IOO et $30 \mathrm{p}$. Ioo.

\section{0) Espagne}

TABI,EAU 2

Pourcentages relevés dans un échantillon composé de six colonies en provenance d'Espagne

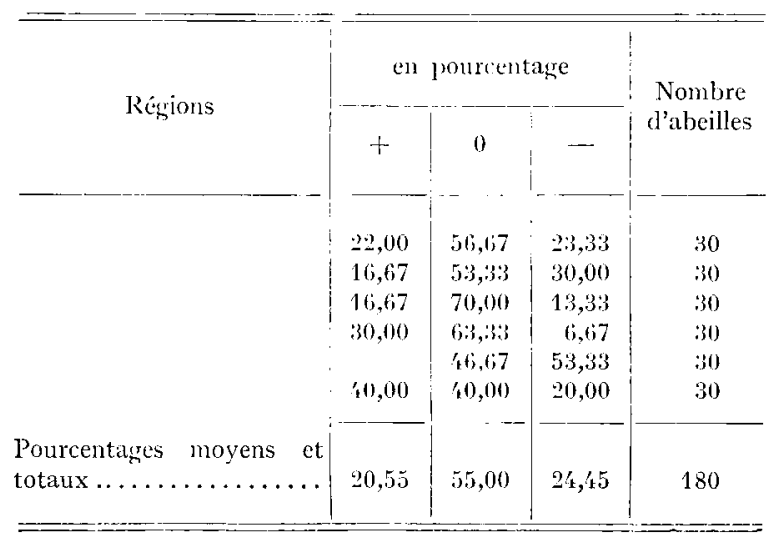


Les pourcentages moyens ne portent que sur I 80 abeilles. Le tableau 2 met en évidence une grande variabilité de la catégorie positive entre ruches (o à $40 \mathrm{p}$. IOO). Ires pourcentages généraux montrent un balancement presque égal du caractère de part et d'autre de la catégorie moyenne.

$3^{\circ)}$ Afrique du Nord

TABLEAU 3

Pourcentages relere's dans un ichantillon conposi de quinse colonies provenant d'Afrique du Nord.

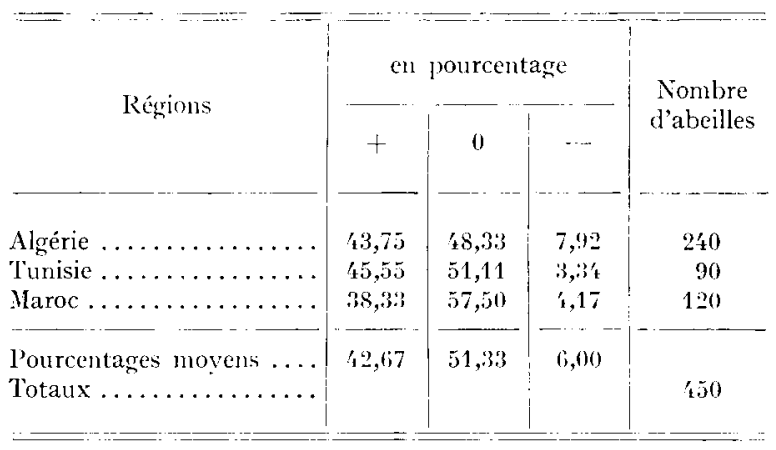

Il ne s'agit plus d'A pis mellifica mellifica mais d'A pis mellifica intermissa décrite par V. BUT'TEL REEPEN. Par rapport aux deux échantillons précédents, celui-ci se caractérise par un assez fort pourcentage de fluctuations positives qui s'élève aux dépens des pourcentages de fluctuations négatives.

$4^{\text {) }) ~ I t a l i e ~ e t ~ L i b a n ~}$

TABLEAU 4

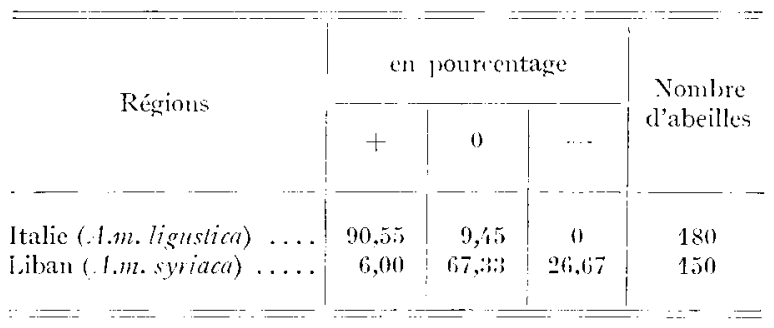

Les deux sous-espèces en provenance de ces régions (. 4 .m. ligustica et $A . m$. syriaca) présentent une couleur très jaune, due à la dépigmentation des téguments des segments abdominaux, ainsi que plusieurs caractères semblables et notanment la pilo- 
sité générale. On pourrait supposer que ces deux formes ont eu dans le passé une origine commune dans la sous-espèce africaine $A . m$. fasciata. Mais ce que nous savons des origines d'A.m. ligustica (RUTTNER I952) et le fait que les types de fluctuations discoïdales soient rigoureusement opposés prouvent que les caractères semblables ne sont que des convergences. On trouve aujourd'hui $A$.m. ligustica sur tous les continents. En ce qui concerne l'abeille française, nous considérons que, dans un échantillon, une tendance vers une fluctuation positive est une preuve de la trace plus ou moins lointaine soit de la sous-espèce A.m. ligustica soit de A. m. carnica. Cet aspect positif de la translation discoïdale constitue une caractéristique commune à ces deux sous-espèces et rigoureusement absolue.

\section{B. - REPRÉSENTATION GRAPHIQUE DES TENDANCES DOMINANTES DE VARIATION DE I,A TRANSIATION DISCOÖDALE}

Les tableaux de chiffres décrivent la variation géographique du caractère mais n'en font pas apparaître les propriétés. Pour tenter de mettre en évidence les aspects cinétiques de cette variation, nous avons recherché différentes méthodes de représentation graphique. Il est apparu que la représentation la plus descriptive est celle basée sur le graphique à coordonnées triangulaires.

Les trois variables sont exprimées en pourcentages, leur total est toujours égal à Ioo. Les valeurs sont portées sur les médianes des triangles par ordre croissant à partir des côtés vers les sommets. Les pourcentages nuls se situent donc sur les côtés. Lorsque dans l'une des trois catégories un pourcentage est égal à roo, la position du point de représentation se confond avec celle de l'un des trois sommets.

Chaque point intègre donc la totalité de l'information; celle-ci a été rectreillie et figurée à des niveaux différents.

$\left.I^{\circ}\right)$ Représentation graphique des tendances dominantes de la translation discoïdale par région (fig. 2)

L'aire délimitée par les points correspondants à chacune des régions géographiques françaises est nettement parallèle aut côté AC (Axe $x$ ). Chacun des points représente une région soit plusieurs ruchers. Les points qui délimitent l'aire de l'axe $\mathrm{Y}$ représentent chacun une muche hybride italienne (RHI). Ces points sont figurés à titre de comparaison en vue de délimiter l'emplacement occupé par ce type de colonies que l'on rencontre fréquemment dans les échantillons, auprès d'autres ruches composées d'abeilles indigènes.

Dans le cas de la figure 2, l'Abeille italienne pure se situe en B. L'échantillon se place au niveau du rucher, c'est-à-dire qu'il porte sur plusieurs colonies.

Chaque point situé de part et d'autre de l'axe $Z$ représente, sauf pour la région des Landes, une analyse portant sur un rucher.

Les hasards des récoltes et des envois d'échantillons ne nous ont pas permis de donner la même valeur à chacun des points. Toutefois le nombre de colonies est toujours plus 
élevé pour les régions géographiquement très voisines (en France), où les formes d'abeilles sont à priori moins différenciées entre elles. Par conséquent, l'information contenue dans chaque point est d'autant plus précise que les échantillons sont géographiquement proches l'un de l'autre par leur origine réelle. Les hybrides avec les

FRANCE

Zône du Cline

- Ruches nybrides italienne

a Alpes

b Jura

c Bretagne 1

d Bassin Porisien

e Midi

Bretogne 2

Massif Central

Normandie 
$\left.2^{\circ}\right)$ Représentation graphique des tendances dominantes de la translation discoïdale par ruche (fig. 3)

Quatre régions ont été représentées par des signes conventionnels différents. Bien que les échantillons soient inégaux en nombre, les zones occupées par chacun d'eux sont nettement visibles.

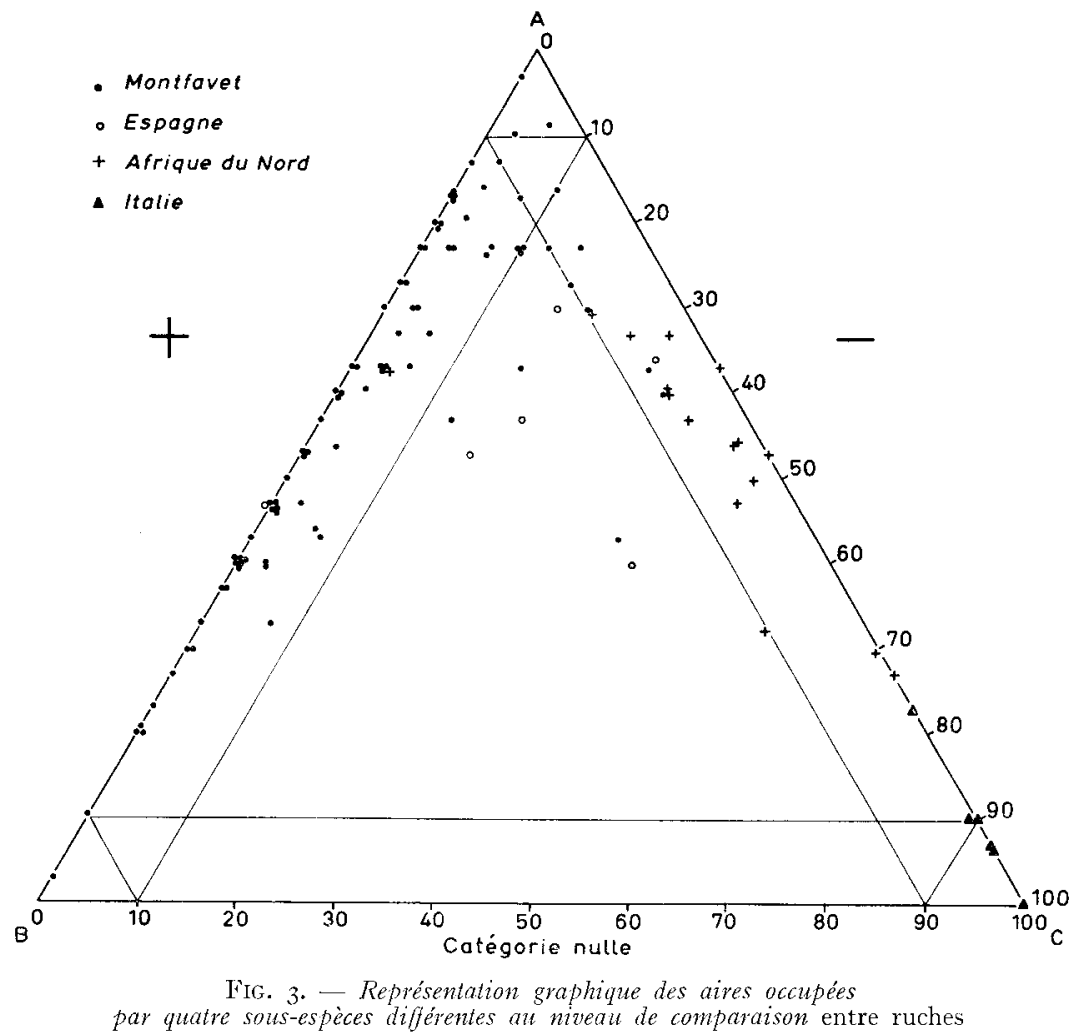

a) France. 86 colonies de provenances diverses et établies depuis quelques années à la station d'Apiculture expérimentale de Montfavet (Vaucluse) se répartissent sur le côté $\mathrm{AB}$ (à gauche de la figure), c'est-à-dire que la variation positive est nulle ou faible.

b) Afrique du Nord. Cette région occupe le côté opposé (AC) : dans ce cas, c'est le type négatif qui est rare.

c) Espagne. Les ruches en provenance de cette région se situent entre les deux zones précédentes.

d) Italie. A.m. ligustica se situe très près du sommet C et présente une fluctuation positive qui peut être totale. 


\section{DISCUSSION}

La discussion porte tout d'abord sur l'ensemble des tableaux et la figure 2 puis la généralisation sur la figure 3 .

\section{A. - IA VARIATION DISCOÏDAIE EN FRANCE}

Le tableau I, les informations complémentaires qui y sont jointes et la figure 2 montrent que :

$\left.\mathrm{I}^{0}\right)$ Les fluctuations de type positif ne sont jamais supérieures à 8 p. Ioo chez 1'abeille indigène ;

$\left.2^{\circ}\right)$ lorsque le seuil de 8 p. Ioo est dépassé, il y a doute possible sur la pureté des échantillons ;

$\left.3^{\circ}\right)$ lorsque le seuil de ro p. Ioo est atteint ou dépassé, il y a certitude que l'échantillon contienne des hybrides italiennes ou carnioliennes.

Nous remarquons que le point France qui résume l'information contenue dans 5452 abeilles se situe précisément au-dessus du seuil de 8 p. Ioo et à la limite de celui de ro p. Ioo. Or, la position de ce point est influencée par les pourcentages recueillis dans les Landes, le Massif Central, l'Alsace-I,orraine et surtout la Vallée de la Loire. Ces régions se situent nettement dans la zone occupée normalement par les ruches hybrides italiennes (RHI) ou au moins pour le rucher de type carnolien au-delà du seuil de Io p. IOO (RHC). La position de chacun des autres points qui représentent le reste de l'échantillonnage d'Abeilles françaises n'apporte pas d'informations complémentaires. Tout au plus, peut-on remarquer un certain regroupement des régions de l'list vers le sommet A, Alsace-Lorraine (?), Jura, Alpes et la position très séparéc de la Normandie. Il n'est pas possible toutefois de tirer d'autres conclusions.

Il faut maintenant apporter quelques restrictions aux trois aspects généraux que nous venons de préciser. In effet, certaines colonies françaises présentent parfois de façon stable un déplacement faiblement positif. Ce déplacement n'est toutefois jamais comparable à celui des sous-espèces importées $A$.m. ligustica et $A$.m. carnica ou à celui de leurs hybrides. Le déplacement est beaucoup plus faible et dépasse de peu l'épaisseur d'une nervure. I1 faut donc distinguer :

Io) la fréquence d'apparition dans chaque catégorie qui, dans le cas présent, quelque soit le sens de la fluctuation est supérieure à 80 p. Ioo,

$\left.2^{\circ}\right)$ l'amplitude du déplacement dans un sens ou dans l'autre. Or nous avons remarqué que lorsque la fréquence est grande dans l'une des deux catégories extrêmes, (supérieur à $50 \mathrm{p}$. Ioo pour l'Abeille française) précisons que ce cas est très rare, le déplacement s'il est positif est faible et le déplacement négatif est fort.

Ces deux tendances opposées ont été rencontrées respectivement l'une en Bretagne, l'autre dans le Midi. Ces deux ruchers étaient isolés depuis plus de dix ans et redevenus presque sauvages. I, tableau 5 montre que dans le cas du second rucher, le fort pourcentage négatif était vraisemblablement le propre d'une colonie et que le caractère s'est transmis probablement par les mâles à deux autres colonies. Nouş 
sommes dans l'impossibilité de conclure à une mutation survenue dans le sens dominant de la variation ou à une sélection qui se serait réalisée dans le même sens. La seconde hypothèse semble confirmée par l'existence de forts pourcentages rencontrés également dans le sens faiblement positif. Mais dans le cas de la translation disco"dale le seuil entre un effet de sélection et une faible mutation n'est pas nettement observable. Quoi qu'il en soit, dans le cas de l'abeille française, nous sommes certains qu'un déplacement positif faible et même se produisant au sein d'une colonie dans Ioo $p$. Ioo des cas, n'est pas forcément l'indication d'une hybridation. D'une part la constance à l'intérieur de la colonie, d'autre part l'absence dans le rucher de toute autre preuve morphologique d'hybridation même lointaine permet de penser qu'il s'agit-là d'une caractéristique rare, mais possible et aisément reconnaissable.

\section{TABLEAU 5}

Cas de variation extrême de type négatif relevé dans le Sud Est de la France

\begin{tabular}{|c|c|c|c|c|}
\hline \multirow{2}{*}{ Origine géographique } & \multirow{2}{*}{$\begin{array}{c}\text { No } \\
\text { d'analyse }\end{array}$} & \multicolumn{3}{|c|}{$\begin{array}{l}\text { Type de variation } \\
\text { en pourcentages (1) }\end{array}$} \\
\hline & & + & 0 & - \\
\hline Noves (Vaucluse). & 83 & 0 & 20 & 80 \\
\hline Noves (Vaucluse) ....... & 84 & 3 & 47 & 50 \\
\hline Noves (Vaucluse) ....... & 85 & 3 & 40 & 57 \\
\hline
\end{tabular}

(1) Calculé sur 30 abeilles par ruche.

Enfin, la région des Landes du Sud-Ouest a fait l'objet d'une étude très approfondie portant sur plus d'un millier d'abeilles. Si par les autres caractères, 1'Abeille landaise s'apparente à l'Abeille française, le pourcentage général de fluctuations négatives est légèrement différent. Nous pensions que le tapis végétal très particulier aurait pu provoquer une hypothétique transformation de la morphologie sous l'in'fluence de certaines caractéristiques de l'alimentation. Celle-ci, en effet, a pour base essentielle les miels d'Erica et de Calluna. Il n'en est rien. Mais nous pensons qu'en ce qui concerne ce caractère (fig. 2 ) :

Io) les transhumances assez limitées vers 1'Espagne,

$2^{\circ}$ ) les importations récentes d'A. $m$. ligustica (bien que les reines aient été en apparence rapidement éliminées) ont eu une action convergente tendant à placer graphiquement cet échantillon en équidistance entre la France et l'Espagne et à le déporter légèrement vers la zone occupée par les hybrides italiennes. Notons au passage que toutes les abeilles non locales s'accommodent très mal des conditions de vie landaises et que si cette abeille subit une différenciation propre depuis quelques siècles, celle-ci n'est pas encore observable autrement que par cette légère élévation du pourcentage de variations positives qui pourrait, par hasard, être liée à des variations plus fines non encore visibles de caractères physiologiques ou de comportement. 
Nous pensons ainsi avoir défini d'une façon aussi précise que possible les aspects de la variation discoïdale de l'Abeille en France.

\section{B. - LA VARIATION DISCOÏDALE EN ESPAGNE ET EN AFRIQUE DU NORD}

Si l'on suit la progression des pourcentages généraux positifs, on remarque que celle-ci se fait de la façon suivante : Io pour la France, 20 pour 1'Espagne, 4o pour 1'Afrique du Nord. La progression des pourcentages généraux nuls est plus constante et varie au plus entre 50 et 60 (France 59,56, Espagne 55,00; Afrique du Nord $5 \mathrm{I}, 33$ ). En arrondissant ces chiffres à I p. Ioo près nous trouvons 60-65-50. L'enrichissement en formes positives s'effectue aux dépens de la classe négative où la progression est inverse (France 30,55 , Espagne 24,45 , Afrique du Nord 6,00). Nous pensons que pour ces trois régions, le pourcentage de formes nulles est constant et que les différences qui progressent de 5 en 5 représentent la part d'interprétation de l'observateur en ce qui concerne les cas litigieux où la position du point discoïdal n'est pas nettement nulle mais très légèrement positive. Nous verrons que la façon de recueillir les données permet de pressentir des écarts pouvant varier avec l'opérateur lorsqu'il s'agit de cas qui ne sont pas nettement définis. Dans le cas présent, ces écarts de 5 p. Ioo que nous pensons devoir attribuer à l'estimation, restent cependant à l'image de la tendance générale. Si l'on joint par une droite (axe $Z$ ) les positions extrêmes c'est-à-dire la France et la Tunisie et que l'on projette sur cet axe les points les plus proches qui se trouvent de part et d'autre de cet axe, on observe la succession suivante: France, Landes, Espagne, Maroc, Algérie, Tunisie. Or, c'est là très exactement le chemin à suivre par les terres pour se rendre d'un point extrême à l'autre. Nous pensons que ce n'est certainement pas l'effet d'un simple hasard qui peut aligner dans l'ordre six points différents et que nous sommes en présence d'un cline.

Von BuTter, REEPEN a décrit avec raison la forme $A . m$. intermissa que nous considérons comme une sous-espèce, mais il semble que la véritable forme intermédiaire soit l'abeille espagnole. D'autres caractères peuvent confirmer cette observation et notamment le nombre de reines élevées spontanément par chacune de ces trois formes d'Abeilles.

L'abeille française peut élever parfois un nombre assez important de reines mais ce nombre est généralement inférieur à Io. Des apiculteurs espagnols dignes de foi nous ont affirmé que chez eux, ce nombre était le plus souvent supérieur à 20 . Chez A.m. intermissa le nombre de cellules royales est voisin de 50 , avec présence permanente de reines vierges parmi la population, ce qui d'ailleurs semble être un point convergent avec les genres Trigona et Melipona tropicaux et néotropicaux. Si cette position de l'abeille espagnole peut être à nouveau confirmée dans l'avenir, nous pensons qu'il serait commode de nommer cette population. Le terme iberica ayant été déjà utilisé pour une forme d'abeille A.m. Caucasica natio iberica par Skorrkov nous proposerions, dans ce cas, $A . m$. hispanica étant donné qu'elle semble bien différer par plusieurs caractéristiques et qu'il ne s'agit ni de la forme mellifica ni d'intermissa. Enfin, s'il n'est pas possible de prouver l'existence d'un caractère particulier existant dans 75 p. Ioo ou roo p. Ioo des cas, cette forme ne pourra être élevée au rang de sous-espèce, mais alors il sera difficile de la rattacher à l'une plutôt 
qu'à l'autre. Le caractère péninsulaire du territoire et de la barrière pyrénéenne auraient pu favoriser dans ce cas la différenciation.

\section{C. - IA VARIation Discol̈DaIE Chez A. Mellifica ligustica ET $A$. Mellifica syriaca}

Nous ne possédons pas d'échantillon provenant de régions géographiquement voisine de celle oì vit l'abeille libanaise, celle-ci se trouve de ce fait isolée au sein de notre étude. Après un examen rapide, l'abeille syrienne semble plus petite et plus " grise ", elle est en tous cas réputée plus agressive que l'abeille italienne, mais par bien d'autres caractères, elle peut paraître assez proche de l'abeille ligurique et notamment par la couleur jaune et la pilosité générale. Or, confirmant les présomptions énoncées par RUTTINER (I952) et solidement basées sur une étude des glaciations en Europe au cours de la période quaternaire, il se trouve que les tendances des fluctuations du point discoïdal ont été, pour ces deux sous-espèces, rigoureusement opposées. Cette comparaison semble done confirmer :

$\left.I^{\circ}\right)$ que l'abeille ligurique n'a pas de parenté avec les abeilles jaunes méditerranéennes orientales ou africaines;

$\left.2^{\circ}\right)$ (qu'elle est apparentée plutôt à une souche originaire de Carniole et de Carinthie.

En effet, la tendance vers une position positive absolue, rapproche la sous-espèce ligustica des deux sous-espèces carnica et banatica, qui présentent la même tendance positive absolue et de nombreux autres caractères notoirement communs. Ceci suggère le regroupement de ces trois sous-espèces au sein d'un exerge. Toutefois, ce rapprochement sera étudié plus en détail ultérieurement. Notons que Rihar (I96I) a montré l'aptitude de la forme carnica noire, à varier de coloration et que la sousespèce banatica semble témoigner encore de nos jours de la possibilité pour deux populations de diverger en deux formes jaunes et noires à partir d'une population ancestrale unique. Le facteur d'isolation de la souche actuelle italienne jaune pouvant être comme pour l'abeille espagnole le caractère péninsulaire du territoire.

\section{D. - GÉNÉRAIISATION}

La discussion de paragraphe porte sur le graphique 3. L'examen de ce graphique montre une zone vide de points correspondants aux valeurs. inférieures à $40 \mathrm{p}$. Ioo dans la catégorie nulle. Nous avions pensé tout d'abord à une anomalie. Un examen plus approfondi montre qu'il ne s'agit que d'une évidence, en effet il n'y a jamais au sein d'une même population un partage égal des pourcentages ou des mesures entre catégories extrêmes. Le balancement du caractère observé ici au niveau des sous-espèces s'effectue, comme toujours, autour de la catégorie moyenne. L'existence de pourcentages égaux ou élevés également répartis entre deux catégories extrêmes serait une anomalie biologique. Celle-ci pourrait peut-être se rencontrer dans le cas, non d'une hybridation mais d'un mélange entre la population d'une colonie de type totalement négatif et celle d'une colonie de type positif. La distribution des données serait bimodale avec des écarts considérables entre les moyennes. Il n'y a 
donc aucune probabilité pour observer l'existence de points sur le côté BC ailleurs qu'en $B$ ou en $C$.

La présence de points dans cette zone serait donc à notre avis la preuve d'un mélange de sous-espèces. Il nous a paru nécessaire de le signaler car un tel mélange peut être effectué par hasard en apiculture, lors de la constitution des essaims artificiels.

Cet aspect graphique du caractère est évidemment commun à tous les échantillons. Il nous paraît inutile de rechercher les moyens d'appliquer des coefficients de correction dans le simple but d'obtenir une figuration moins dense et mieux répartie et qui serait dans tous les cas non conforme à la réalité.

\section{E. - INTERPRÉTATION DE LA TRANSLATION DISCOÏDAIE EN FONCTION DE IA MORPHOLOGIE ALAIRE GÉNÍRALE}

\section{$\left.\mathbf{I}^{0}\right)$ Considérations sur la méthode employée pour recueillir les données}

Si l'on se reporte à la figure I, on remarque que les zones positives et négatives sont linéaires alors que la zone nulle est ponctuelle. Or la probabilité pour qu'un point dont la position varie linéairement entre en coïncidence exacte avec un autre point de cette ligne est faible. Ce qui revient à dire que statistiquement on a beaucoup plus de chance de trouver des positions positives ou négatives que nulles. Dans ce cas, les différences que l'on est en droit de supposer apparaîtront au profit des zones extrêmes et aux dépens de la zone nulle. Ceci peut alors entraîner les deux conséquences suivantes.

Io) Selon l'opérateur et. selon la fréquence des cas où la position nulle est douteuse, on peut enregistrer des écarts entre les pourcentages des différentes catégories.

$\left.2^{\circ}\right)$ Ces erreurs d'estimations cumulées avec l'effet statistique théorique ci-dessus exposé auraient pour conséquences de sensibiliser les zones extrêmes au détriment de la zone intermédiaire qui serait alors plus importante qu'elle n'est en réalité.

Bien que le raisonnement paraisse logique, on ne peut améliorer que faiblement la méthode actuelle qui semble satisfaisante. Par ailleurs, toutes les estimations ont subi les mêmes effets. Néanmoins, afin que les travaux puissent être reproduits ultérieurement par les différents auteurs dans des conditions aussi semblables que possibles et que par là les résultats soient aisément comparables quel que soit 1'expérimentateur, il serait possible d'admettre une zone nulle non ponctuelle. Toute fluctuation qui se situerait de part et d'autre de l'axe de référence dans une limite n'excédant pas l'épaisseur d'une nervure, pourrait être également considérée comme nulle. Bien entendtu, une étude statistique effectuée à partir de mensurations et non d'estimations, permettrait de déterminer une zone de fréquence maximum autour de cet axe, et de préciser les limites de trois zones égales. L’intérêt présenté par le caractère d'une part, les causes d'erreurs que nous préciserons plus loin et auxquelles il est soumis d'autre part, ne justifient pas une telle précision. Cette méthode ne ferait qu'accroître les difficultés rencontrées lors du recueil des données. Il paraît plus souhaitable d'admettre la méthode simple actuelle qui permet d'observer un plus grand nombre d'individus avec moins de précision. 


\section{$\left.2^{\circ}\right)$ Étude des relations probables entre la variation du point discoïdal et la variation des points qui servent à la détermination de l'axe de références vertical}

Io) Technique générale.

Les mensurations effectuées sur l'aile des insectes montrent que les valeurs se distribuent de part et d'autre d'une valeur moyenne avec une fréquence déterminée et parfaitement calculable. Les tronçons de nervure ont donc pour chaque individu une valeur propre exprimable par $\bar{m} \pm \varepsilon$. Il s'ensuit qu'aucune aile n'est rigoureusement superposable à une autre puisque chaque point de convergence des nervures peut prendre une valeur indépendante de l'ensemble de l'aile, mais fort heureusement entre certaines limites seulement.

La lecture des travaux de MAGNAN (I934) nous a remis en mémoire un procédé utilisé par cet auteur et qu'il expose ainsi :

" Si on projette un dessin d'aile à l'aide d'un appareil à projection, on obtiendra une figure plus ou moins grande et on peut s'arranger de façon que telle longueur caractéristique... ait exactement une valeur fixée à l'avance. "

Nous avons simplifié cette méthode en projetant une première aile d'abeille sur une feuille de papier blanc, à l'aide d'un agrandisseur photographique. On en relève le croquis. Puis, on applique exactement le même grossissement à une seconde aile dont la silhouette vient plus ou moins en coïncidence avec le croquis relevé de la première. Pour que les deux ailes soient apparemment superposables, il suffit de rester en deçà de la valeur moyenne de variation $\pm \varepsilon$ lors de la projection. Les figures 4 et 5 ont ainsi subi un agrandissement $\times 20$. On peut utiliser cette méthode de comparaison dans deux cas différents.

a) A l'intérieur d'une même espèce. - On applique exactement le même grossissement aux deux silhouettes ce qui permet d'apprécier d'un seul coup d'œil les variations de taille. Sur la figure 5 , on remarque ainsi que 1'abeille bretonne présente une aile plus grande que l'abeille méđiterranéenne française. Il en est de même de l'aile de 1'abeille italienne qui est plus grande que 1'aile de l'abeille française moyenne. Cette comparaison peut s'étendre à chaque tronçon de nervure. Mais on peut également noter toutes les variations de formes des cellules; ainsi sur la figure 4 on remarque nettement la forme en haltère de la $3^{\mathrm{e}}$ cellule cubitale signalée déjà par GoETZE, ainsi qu'un allongement de la $2^{\mathbf{e}}$ cellule discoïdale. Les meilleurs points de repère mis en coïncidence pour effectuer ces comparaisons nous ont paru être :

I) le bord supérieur de l'aile ;

2) la nervure basale;

3) la $2^{\mathrm{e}}$ nervure transverso-cubitale.

b) Entre espèces différentes. - Quelle que soit la taille de chacune des espèces, on recherche alors la meilleure coüncidence entre les surfaces en faisant varier le grossissement. Dans ce cas, ce sont surtout les modifications de formes des cellules qui présentent un intérêt pour l'étude de l'évolution propre de l'aile.

Dans les deux cas, le procédé de comparaison est rapide, aussi sensible qu'on le désire et évite la longue recherche numérique par sondage et mensuration des éléments supposés variables. 


\section{$\left.2^{\circ}\right)$ Application à l'étude de la translation du point discoïdal.}

Considérons tout d'abord le cas le plus général de la variation correspondant type nu1. La figure 5 fait apparaître que la rétraction générale de la taille de l'aile ne semble pas influer sur l'équilibre d'ensemble de la nervation ; d'autre part quelle que soit la taille de la cellule radiale, celle-ci n'influe pas sur la position de la droite AB qui apparaît comme un excellent axe de référence bien que la longueur puisse varier dans les limites $\mathrm{BB}^{\prime}$ ainsi que la largeur.

Dans le cas de la position positive, l'interprétation est complexe, la figure 4 a été établie d'après plusieurs croquis, et met en évidence plusieurs types de variations de l'aile de l'abeille italienne.

a) déplacement de l'axe du nervulus (ce caractère est trop variable et ne peut être considéré comme descriptif),

b) augmentation de l'angle formé par le coude de la $2^{\text {e }}$ nervure récurrente $(\mathrm{E} G$ ) et déplacement de son point de contact $(\mathrm{G})$ sır la base de la $3^{\mathrm{e}}$ cellule cubitale ; la variation de l'index cubital peut donc être influencée à la fois par ce déplacement et par celui de $\mathrm{F}$ en $\mathrm{F}^{\prime}$,

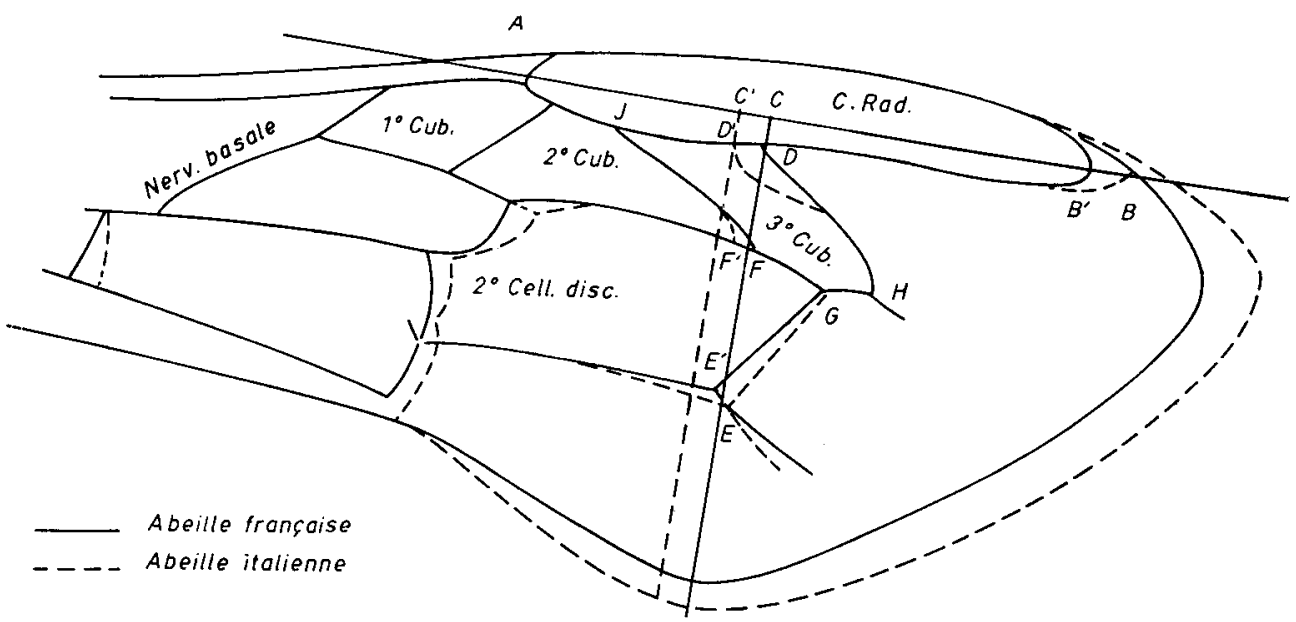

FIG. 4. - Application de la technique de projection avec grossissement constant tendant à montrer les principales variations de forme des cellules chez $\mathrm{A}$. $\mathrm{m}$. ligustica SPIN.

c) il s'ensuit que l'index cubital $\mathrm{FG} / \mathrm{GH}$ peut être influencé également par la variation $\mathrm{FF}^{\prime}$ mais aussi par la variation propre à $\mathrm{GH}$.

d) la variation ayant le plus de conséquences sur l'appréciation de la position du point discoïdal est le déplacement de $\mathrm{D}$ en $\mathrm{D}^{\prime}$.

Il apparait, en effet, que si l'axe $A B$ n'est pas ou peu variable, il n'en est pas de même de la perpendiculaire $\mathrm{CE}$ car si chez l'abeille italienne la position du point $\mathrm{E}$ est à Ioo $\mathrm{p}$. Ioo positive, c'est, certes, à cause d'un allongement relatif de la $2^{\mathrm{e}}$ cellule discoïdale mais aussi d'un report fréquent de $\mathrm{D}$ en $\mathrm{D}^{\prime}\left({ }^{1}\right)$ qui rend le dépla-

(1) En I959, GoFTzE a signalé une relation entre JD/FH et le type de variation discoïdale ; cette relation peut confirmer la présente observation, car elle introduit la position du point $\mathrm{D}$ dans ce qu'il nomme " l'Hantel index". 
cement plus important qu'il n'est en réalité car on remarque que si l'axe CE était élevé en $C$, le point $E$ serait très faiblement positif quant à $E^{\prime}$ par rapport au même axe il n'est que très faiblement négatif. En fait, le balancement positif ou négatif du point discoïdal par rapport à l'ensemble de l'aile existe mais semble beaucoup plus faible que l'on pourrait le penser. L'application de la technique de superposition semble donc permettre :

I) de préciser certaines causes de la variation de ce caractère. Celui-ci n'avait été jusqu'à présent qu'observé et utilisé d'après des données apparentes ;

2) de ramener les valeurs de la variation à de plus justes proportions tout en confirmant l'existence de deux balancements, l'un réel et l'autre apparent. (Le déplacement de $\mathrm{D}$ en $\mathrm{D}^{\prime}$ corrélatif au déplacement $\mathrm{EE}^{\prime}$ constitue une propriété de 1'aile A.m. liguistica que l'on ne retrouve pas par exemple dans la figure 5.)

3) de préciser les limites entre lesquelles ce caractère peut être utilisé et la confiance que l'on peut accorder à sa valeur descriptive dans la discrimination morphologique des différentes formes d'A. mellifica.

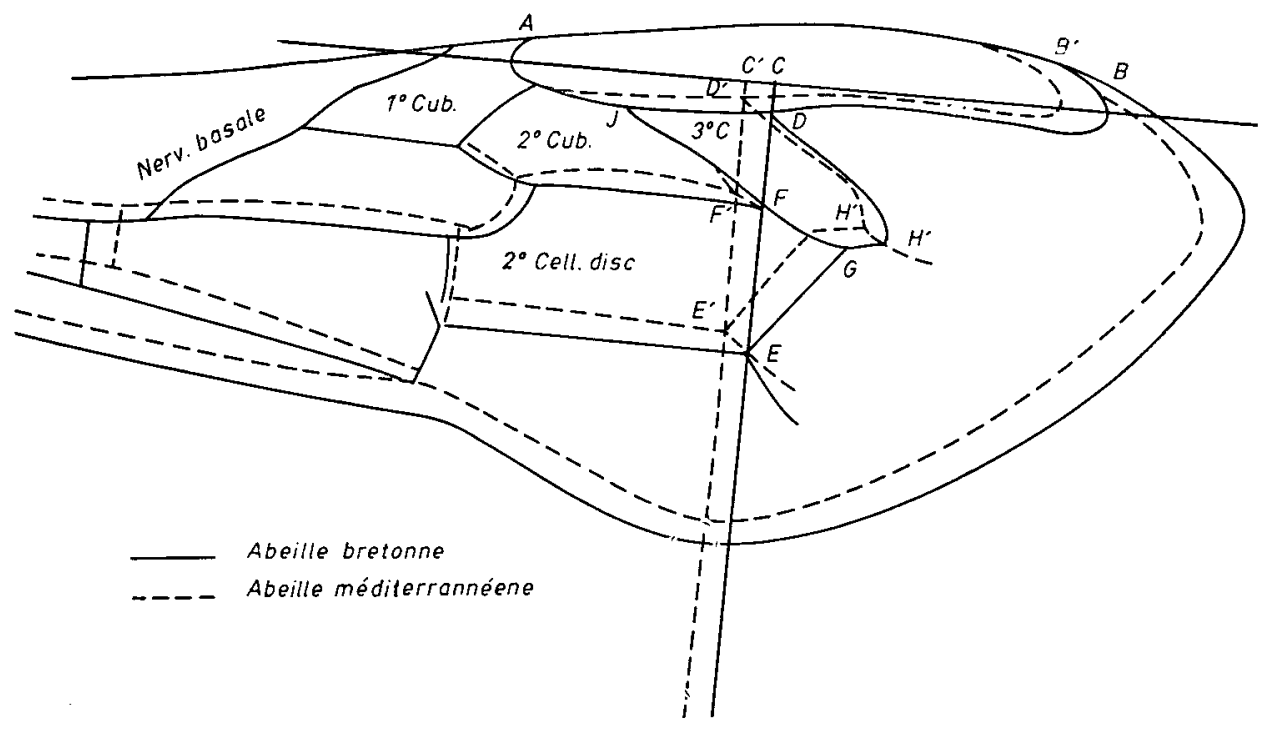

FIG. 5. - Application de la technique de projection avec grossissement constant tendant a montrer les différences de tailles entre les ailes sans modification de forme des cellules

\section{CONCLUSIONS}

La présente étude de la variation du point discoïdal permet donc de penser :

$\left.\mathrm{I}^{0}\right)$ que la quantification de ce caractère est difficilement concevable autrement que par pourcentages, statistiquement peu maniables;

$\left.2^{0}\right)$ que la conception actuelle du recueil des données peut tendre vers une sensibilisation de la distribution vers les zones extrêmes positives et négatives au détriment de la catégorie moyenne. 
Cette sensibilisation semble cependant pouvoir être admise à condition de n'être qu'un moyen de mettre mieux en évidence des phénomènes observés sur un grand nombre de cas, surtout lorsque les échantillons proviennent de régions géographiquement rapprochées ;

$\left.3^{\circ}\right)$ qu'un des axes de références supposé stable est soumis à une variation propre tendant encore à exagérer le balancement du caractère entre des limites qui ne sont pas en rapport avec la variation de l'ensemble de la nervation;

$\left.4^{\circ}\right)$ que le caractère tel qu'il a été défini par GOETzE, tel qu'il est utilisé, permet une discrimination variétale très intéressante, qu'il présente un intérêt taxonomique certain, qu'il y a lieu cependant de ne pas omettre les facteurs éventuels de variation mis en évidence dans la présente note lors de 1'interprétation des réstrltats.

$$
\text { Reçu pour publication en juillet } 1963 .
$$

\section{SUMMARY}

STUDY OF THE DISCOIDAL-POINT TRANSLATION IN THE WING OF BEES

The author describes different aspects of a morphological characteristic of the wing, which GOETZE had previously termed "Discoidalverschiebung ". The different types of fluctuation of the discoidal point are considered as characteristics of subspeciation applicable to the study of variants of the species $A$. mellifica $\mathrm{L}$. in specimens of French and Mediterranean origin. The kinetic aspects of the variation in this characteristic are explained by the application of a method of graphical representation along triangular co-ordinates. Having shown the existence of morphological clines, the author devotes the latter part to a critical study of the method. After suggesting a slight modification for assuring better balance in the assessment of the data, he proceeds to an attempt at specifying the elements of the wing structure which can influence the variation in the characteristics under consideration.

\section{RÉFÉRENCES BIBLIOGRAPHIQUES}

Bernardi G., 1957. Contribution à l'étude des catégories taxonomiques : 1. Avant propos, nomenclature et définition. Bull. Soc. Ent. France, 61, 194-200.

II. Les règles internationales de nomenclature zoologique et la notation des catégories taxonomiques. Bull. Soc. Ent. France, 62, 224-250.

Boquet Ch., 1953. Thèse. Arch. Zool. Exp. Gén., 90, 187-450.

GoETzE, 1959-1960. Welche Merkmale eignen sich zur Rassenteurteilung im Körverjahren. Siudwestdeutscher Imker, 1959, (I o) 229-230; (I I) 268-269; (I 2) 300-301 ; I960 (I) I0-I I I I I-I 2 I 65 -I 66.

GoETzE, I 959. Die Bedentung der flügelgläders für die Zuchterische Beurteilung der Honigbiene. Z. Bienenforsch., 4, I4I-I 48.

Huxley J,, 1938. Cline, an auxiliary taxonomic principle. Nature, 3587, 219-220.

HuXley J., 1939. Clines, an auxiliary method of taxonomy. Bijdr. Dierk., 27, 49I-520.

Huxley J., 1940. Towards the new systematics. In. The New syslematics, Oxford, I-46.

HUXLEY J., I942. Evolution, the modern synthesis. London, 645 p.

HuXley J., I955. Morphism and Evolution. Heredity, 9, I-52.

MAA T., I953. An inquiry into the systematics of the tribus Apidini or honeybees (Hymen.) Treubia, 21, 525-640 (Java).

Magnan, I 934. Le vol des Insectes. Paris.

MAYr E., I942. Systematics and the origin of species. New York, I-XIV, 334, 29 fig.

RutTner F., I952. Alter und Herkunft der Bienenrassen Europas. Öterreischer Imker, s. p. I-4. 
Rihar, i96r. Recherches biométriques sur la couleur des Abeilles carnioliennes en Yougoslavie. Ann. Abeille, 4, I-5o.

Teissier, I962. Dynamique des populations et Taxonomie. Ann. Soc. Roy. Zool. Belgique, 83, 23-44.

VERITY R., I925. Remarks on the evolution of the Zygaenae and an attempt to analyse and classify the variations of Zygaena lonicerae Scheven and of Zygaena trifolii Lsp. and other subspecies. Ent. Res., 37, IOI-I 40, I I 7-I 2 I, I 35-I 38 , I 54-I 58.

VERITY R., i926. The geographical and seasonal variation of Coenonymphe pamphilus. Zeits. wiss. Insektenbiol., 21, I9I-208.

VERITY R., I929a. The asiatic origins of the western Palseartic Rhopalocera exemplified by Melitaes didyma Esp. Ent. Rec., 41, 31-34, 39-43, 63-67, 72-76, 89-9 I, I I I-I I 8, I 22-I 32.

VERITY R., I $929 b$. Essai sur les origines des Rhopalocères européens et méditerranéens et particulièrement des Anthocaridi et des Lycaennidi du groupe d'Agestis Schiff. Ann. Soc. Ent. France, 98, 323-360.

Verity R., I938-1939. Supplement to the Butterfly races and Zygaena of Macedonis. Ent. Rec. Suppl., 40, I-16; 51, I $7-20$.

Verity R., I940. A revision of the athalia group of the genus Melitaea Fabr. Transp. R. Ent. Soc., London, 89, 89, 59I-702, pl. I-I 4. 